\title{
El desarrollo del concepto de familiaridad de marca y su comprensión actual como variable moderadora
}

\section{The development of brand familiarity concept and its current understanding as a moderating variable}

\section{Lizardo Vargas Bianchi. Universidad de Piura}

Resumen:

El artículo expone el tránsito que ha seguido el concepto de familiaridad de marca desde que fue abordado en la década del setenta, su definición técnica en el campo de las comunicaciones de marketing y los estudios del consumidor, hasta su comprensión y uso actual. Para ello, se ha revisado la literatura publicada en las revistas académicas entre 1970 y el 2010. El estudio observa que, en los últimos decenios, la investigación ha abordado el término desde una perspectiva de procesamiento de la información, particularmente en el efecto moderador de la familiaridad de marca en distintos esfuerzos cognitivos y perceptuales.

Palabras clave:

Familiaridad de marca, procesamiento de información, comportamiento del consumidor

Abstract:

The paper presents a literature review of brand familiarity concept, from its early mentions during the seventies, the technical definition given to it within marketing communications and consumer studies, and its current understanding and usage. The review runs from 1970 to 2010, covering mayor scholarly publications. It is observed that in the last decades familiarity has been researched from an information processing stand point, with a particular focus on its moderating effect in individuals' cognitive and perceptual efforts.

Keywords:

Brand familiarity, information processing, consumer behaviour 


\section{Introducción}

Durante las últimas cuatro décadas, diversos estudios han considerado a la familiaridad como una medida adecuada para tener información sobre la situación de una marca, tanto en la mente de los consumidores como en el mercado y respecto a los productos competidores.

De esta manera, se ha observado su utilidad como un indicador, del mismo modo que sucede con otras variables propias de la comercialización: recordación espontánea y asistida de la marca, su nivel de relevancia, el grado de awareness, goodwill hacia el producto, entre otros.

A pesar de que el significado del concepto ha sido definido como un término técnico de las comunicaciones de marketing, desde hace relativamente poco tiempo se ha manifestado un aumento en la literatura académica que cubre los diversos aspectos de la familiaridad.

Este artículo es una revisión de la literatura, que intenta mostrar el recorrido seguido por el concepto de familiaridad, desde cuando sólo era un adjetivo utilizado de modo genérico para describir la situación de una marca, hasta transformarse en un tecnicismo con sentido propio. Siguiendo ese fin, cabe indicar que la investigación comprende el término familiaridad en el sentido de familiaridad-hacia-una-marca.

Distintas investigaciones realizadas en el campo de la psicología cognitiva facilitaron el marco de estudio y la comprensión de la familiaridad. Este campo de la psicología permitió analizar la influencia de esta variable en los procesos psicológicos del consumidor, en particular el proceso de resolución de problemas y toma de decisiones. Ambos aspectos de alta relevancia en cuanto a las comunicaciones de marketing, entre cuyos objetos se encuentra la comprensión y participación en la decisión de compra.

Cabe mencionar a dos autores en particular. Krugman (1965) introdujo el concepto de la implicación, el envolvimiento o involucramiento (involvement). Ello le permitió explicar que, en determinados escenarios, los individuos procesan la información aún cuando los niveles de atención en los que se encuentran son notablemente bajos. Sus estudios se centraron en la correlación entre la naturaleza de la información expuesta y el grado de importancia que el individuo manifestaba, de acuerdo con lo cual podía determinar su grado de compromiso o envolvimiento con el mensaje.

Por su parte, Zajonc (1968) observó que la simple exposición de un objeto en circunstancias de bajo nivel de atención podría ser una condición suficiente para originar un esfuerzo de procesamiento de información. Asimismo, la exposición repetida del estímulo originaba respuestas afectivas y evaluativas hacia el mismo. Esta afirmación era consistente con principios ya conocidos, como la reducción de la incertidumbre (Harrison, 1977) y la teoría de la fluidez perceptiva (Jacoby, 1991). 
Ambos estudios permitieron comprender mejor el accionar de variables psicológicas de participación sutil (menos intrusivas que la persuasión directa, o reclamo inmediato de acción). Este hecho facilitó investigaciones posteriores de otros elementos influenciadores en la conducta de naturaleza similar, como es el caso de la familiaridad.

Desde sus primeras menciones en la literatura del marketing, la publicidad y la psicología del consumidor, el término familiaridad ha mantenido su presencia y ha sido objeto de observación desde diversos ángulos, hechos que han permitido a los distintos autores no sólo delinear definiciones, sino también describir su accionar en las comunicaciones de marketing.

\section{Metodología}

Atendiendo al objetivo de este artículo, la revisión y compendio de estudios realizados a lo largo de los años sobre la familiaridad de marca, se ha adoptado una metodología de análisis de la literatura científica. Para ello, ha sido necesario examinar las principales publicaciones académicas sobre las comunicaciones de marketing y la psicología del consumidor. El análisis comprende de los años 1970 hasta el 2010.

A pesar de que la palabra 'familiaridad' está contenida en distintos artículos, ya sea dentro de sus textos, o en sus titulares o como parte de los abstracts o palabras clave, sólo se han analizado aquellos relacionados de forma directa con el concepto estudiado. Las principales publicaciones consultadas, en función al volumen de artículos que han incluido en sus colecciones sobre el objeto de estudio del presente artículo, fueron:

Advances in Consumer Research

Journal of Advertising

Journal of Consumer Psychology

Journal of Consumer Research

Journal of Marketing

Journal of Marketing Comunications

Journal of Marketing Research

Esta revisión de la literatura incluye también referencias de otras publicaciones académicas. Si bien el número de artículos sobre familiaridad en sus colecciones es menor, no resta que sus contenidos se consideren relevantes para el cuerpo de conocimiento sobre familiaridad. Estas publicaciones fueron: 
Applied Cognitive Psychology

International Marketing Review

Journal of Business and Psychology

Journal of Business Research

Journal of Marketing Theory and Practice

Journal of Product and Brand Management

Marketing Letters

Psychology and Marketing

La metodología para la revisión de la literatura ha procurado seguir un orden cronológico, con el objeto de identificar la agregación del conocimiento sobre la familiaridad y este efecto sobre el desarrollo del término.

En consecuencia, la revisión se halla dividida en tres secciones. La primera cubre la etapa entre 1973 y 1985 , tiempo en el que las experiencias de consumo y el conocimiento de los productos son los únicos elementos que se vinculan con la familiaridad de marca.

Una segunda etapa, que va de 1986 a 1991, cuya importancia redunda en el hecho de que por primera vez un grupo de autores ofreció una definición técnica de la familiaridad y su relación con las marcas.

Por último, la tercera etapa, que abarca desde 1992 hasta el 2010. Durante este lapso la familiaridad, en una gran parte de las investigaciones, se enmarca en el campo de la psicología del consumidor. De esta forma, el grueso de los estudios la abordan como un agente moderador en el procesamiento de la información y toma de decisión.

\section{Los primeros estudios y la aproximación al concepto}

La primera referencia a la familiaridad se encuentra en Bogart y Lehman (1973), quienes sugirieron que ésta era una meta deseable para cualquier marca. Los autores observaron que la aceptación y la preferencia hacia la marca son características que la familiaridad manifiesta, con correspondientes consecuencias positivas: ventaja dentro de la categoría en que compite, facilitando la predisposición a la compra. 
A pesar de su visión positiva respecto a la familiaridad, señalaron que ésta se encontraba en alta correlación con la cuota de mercado del producto, pero que no era una medición adecuada para definir la posición de la marca entre la competencia y, como consecuencia, tampoco una meta de marketing en sí misma.

De esta manera, Bogart y Lehman (1973) concluyeron que los productos comprados con mayor frecuencia y beneficiados con un fuerte apoyo publicitario gozaban de una mayor familiaridad, a pesar de que dicha medida no necesariamente se vería reflejada en el índice de ventas.

El uso de la familiaridad como herramienta de métrica comercial adquirió, en estos autores, un papel secundario en su estudio, debido al uso primario y generalizado del awareness como la variable dominante de las mediciones. Es probable que aquello ocurriese debido a que el awareness era mejor reconocido en la literatura como una de las mediciones estándar para determinar la posición de la marca en el mercado.

Finalmente, Borgart y Lehman (1973) no profundizaron en la diferenciación de la familiaridad de otros conceptos similares como goodwill (buena voluntad hacia la marca) o reputación, aspectos que benefician a la marca pero, al ser intangibles, difíciles de ser medidos y mejor estudiados.

Poco tiempo después, Politz (1975) sostuvo que la familiaridad originada por la publicidad hacia la marca era una condición suficiente para originar confianza hacia la misma, y, de esta manera, contribuir a las ventas del mismo. El autor se concentró en los efectos psicológicos de la publicidad antes que en sus efectos conativos, hecho que favoreció el origen de lo que denominó 'Principio de Familiaridad'. A pesar de que no definió este principio de forma estucturada, subyace en su lógica el principio de confianza que se origina de modo natural hacia los objetos conocidos.

Algo similar se observa en el artículo de Monroe (1976), sobre la relación entre la familiaridad, el precio y la preferencia de compra. El autor traza una equidad entre la familiaridad y el conocimiento o la experiencia que el individuo tiene con la marca. Su propuesta concluye que la variable en cuestión, en cuanto información/conocimiento del producto, tiene un efecto positivo sobre la decisión de compra.

Los autores Park y Lessig (1977) también situaron sus estudios sobre la familiaridad en un campo cognitivo. Su aproximación al concepto siguió la ruta trazada por las propuestas de Howard y Sheth (1969) sobre las etapas de evaluación y compra en las que se encuentra un consumidor y los criterios de elección que se originan de esas etapas. Éstas son: Solución Extensiva de Problemas (SEP), Solución Limitada de Problemas (SLP), Solución Rutinaria de Problemas (SRP) y Aburrimiento o Solución Exploratoria de Problemas (SEP).

En la SEP, el consumidor básicamente no se encuentra familiarizado, de tal forma que tiene un criterio de selección empobrecido y carece de la habilidad para seleccionar la marca apropiada que satisfaga su necesidad. 
En el caso de la SLP, el individuo ha adquirido unos criterios de selección mejor definidos y su elección de compra es la más apropiada.

En la SRP, el consumidor tiene un mejor criterio de selección y no percibe la ambigüedad entre las marcas; como consecuencia, hay menos marcas dentro de su conjunto de selección (consideration set; Hauser, Toubia, et al., 2010). Howard y Sheth (1969) sugirieron que el grueso de las compras suceden bajo estas características.

En la SEP, la persona tiene suficiente experiencia y se ha adecuado a un patrón de consumo regular y adecuado a sus requerimientos. Es el caso de las compras por impulso, o que acarrean pocos riesgos personales o económicos.

Park y Lessig (1977) registraron que cuando mejor es el criterio de selección se observa un mayor índice de familiaridad hacia la marca. De alguna forma es viable afirmar que el criterio-de-selección constituye un marco de aprendizaje. Por ejemplo, la etapa de la SLP se corresponde con un estado de procesamiento de baja relevancia lo que, en consecuencia, equivale a un estado de pre-aprendizaje; en tanto, la SEP (Solución Limitada de Problemas) se corresponde con una situación de alta relevancia y un estado de mayor aprendizaje.

El consumidor, que puede hacer rutinarias sus decisiones de compra, es una persona que ha logrado un alto grado de destreza respecto al uso y al conocimiento del producto. En ese sentido, la perspectiva de Howard y Sheth (1969) sobre la familiaridad fue vincularla con los niveles de procesamiento de los individuos, enmarcando su estudio en la psicología cognitiva aplicada al marketing.

Algunos años más tarde, los mismos autores publicaron un nuevo artículo sobre la familiaridad (Park y Lessig, 1981). En esta nueva entrega, introdujeron una distinción entre el vínculo familiaridad-consumidor, basándose en el grado y la clase de conocimiento que el individuo tuviera hacia la marca.

Su primera propuesta (Park y Lessig, 1977) se orientó a medir la familiaridad del producto en términos de cuánto sabe la persona sobre el producto. El estudio publicado en 1981 se orientó a medir la familiaridad en términos de cuánto cree la persona saber sobre el producto. Los autores llamaron a la primera "familiaridad objetiva" y a la segunda "familiaridad subjetiva".

Esta distinción fue la primera realizada respecto al concepto de familiaridad, al describir un suceso que ocurre en el mismo individuo pero observado desde puntos de vista diferentes. Los autores también presentaron el conocimiento y la familiaridad de alguna forma relacionadas con la cantidad de información procesada por un individuo. Asimismo, señalaron que comprendían la información no sólo proveniente de fuentes externas, sino también la originada por la experiencia del uso continuado de un producto. 
En ese trabajo, Park y Lessig (1981), hicieron notar la ausencia en la literatura académica de una propuesta de definición conceptual sobre la familiaridad aceptada de forma común.

En el mismo año, Marks y Olson (1981) propusieron acotar la comprensión de la familiaridad de acuerdo con las estructuras cognitivas del consumidor, en particular, aquellas vinculadas a las experiencias pasadas con el uso de productos.

Su trabajo consolidó varias de las ideas sugeridas hasta ese momento por los investigadores en torno a la destreza (expertise), como conocimiento originado a partir de la utilización sucesiva de un producto. En consonancia con esta propuesta, y la de autores antes mencionados, Conover (1982) sugirió que la perspectiva cognitiva era la adecuada para continuar los estudios relacionados a la familiaridad de marca.

Johnson y Russo (1984: 548) introdujeron un nuevo punto de vista cuando señalaron que la familiaridad hacia un objeto influenciaba en el procesamiento de nueva información referida al mismo. En virtud a esta observación, determinaron que la familiaridad poseía una función moderadora sobre el conocimiento de la persona.

En su estudio, los investigadores demostraron que el conocimiento previo aumenta la habilidad del consumidor de codificar y rememorar nueva información. Con esta mejora en la habilidad codificadora, los consumidores familiarizados desarrollan una estructura cognitiva mejor organizada para asimilar nueva información referente al objeto (la marca o producto).

Al observar la influencia de la familiaridad en el procesamiento de la información, reafirmaron el ángulo de estudio del concepto en el campo de la psicología cognitiva aplicada al consumidor, hecho consistente con las afirmaciones previas ya citadas. Tal es así que Johnson y Russo (1984) llegaron a sostener que el mismo conocimiento del producto es sinónimo de familiaridad.

Los citados autores identificaron tres ventajas que un producto y sus consumidores obtienen cuando alcanzan altos niveles de familiaridad.

a) Conocimiento superior de las alternativas existentes. Los consumidores con mucha familiaridad tienden a conocer detalles sobre las alternativas existentes.

b) Mejores búsquedas de alternativas. Los consumidores más familiarizados pueden manejar mejores elementos de comparación entre las diversas posibilidades de una misma categoría de productos.

c) Mejorar el procesamiento de información de productos nuevos y otros ya existentes. Los consumidores más familiarizados pueden utilizar su conocimiento sobre la categoría para enfocar su atención a la información que resulte relevante para la elección de la compra. 
Como se puede observar, las tres ventajas se encuentran vinculadas con la información que el individuo tiene y utiliza en su relación con un determinado producto o categoría de producto. Johnson y Russo (1984) fueron los primeros en continuar la tendencia de Park y Lessig (1981), de enlazar la familiaridad con el procesamiento de información y, por tanto, con el conocimiento.

Este plano de estudio empezó a definir un cuerpo de conocimiento común alrededor del concepto de familiaridad. Zaichowsky (1985) continuó el acuerdo entre la familiaridad y el conocimiento, y sugirió (basado en Bettman y Park, 1980) que la experiencia de uso del producto era la fuente de información más importante en la creación del constructo familiaridad-conocimiento.

A pesar de que los estudios se encontraban acotados, y en acuerdo común en diversos aspectos, hasta ese momento ningún autor entregó una propuesta de definición de familiaridad.

\section{La definición de la Familiaridad}

Baker, Hutchinson et al. (1986) publicaron un artículo sobre la relación entre la familiaridad y los grupos de consideración de marca que supuso un avance importante en el campo.

El autor y sus colegas sugirieron que la familiaridad hacia la marca es, a pesar de su simplicidad, un estado conveniente para mejorar la valoración de un producto en el mercado. "La familiaridad de marca es condición suficiente para aumentar las actitudes hacia la marca así como la elección hacia la misma” (Baker, Hutchinson, et al., 1986: 640).

Pero quizá, el aporte más relevante fue su propuesta de definición de la familiaridad de marca, haciendo que la afirmación de Park y Lessig (1981), sobre la ausencia de una definición aceptada de forma común, quedara obsoleta. Los autores sugirieron lo siguiente: "La familiaridad es una construcción unidimensional que se encuentra directamente relacionada a la cantidad de tiempo que se ha pasado procesando información sobre la marca, con independencia del tipo de contenido que haya sido procesado" (Baker, Hutchinson, et al., 1986: 640).

Baker y colegas (1986) fijaron un hito en la consolidación de la familiaridad como concepto, al ofrecer una base para futuros estudios al respecto en el ámbito del marketing y de las comunicaciones de marca.

Posteriormente, la investigación de Alba y Hutchinson (1987) utilizó la definición de Baker, Hutchinson, et al. (1986) y ofrecieron una segunda y más concisa visión sobre la familiaridad, siempre enmarcada en el terreno de la cognición y la conducta del consumidor. Señalaron que ésta se determinaba por "el nú- 
mero de experiencias relacionadas con el producto que el consumidor ha acumulado". (Alba y Hutchinson (1987: 411)

En ese mismo estudio, Alba y Hutchinson (1987) aportaron una distinción entre la familiaridad y la destreza (expertise). Los autores comprendieron la destreza como una habilidad de realizar bien una labor relacionada con el producto. Desde su perspectiva, la familiaridad es un estado cognitivo, mientras que la destreza es un estado de acción.

Al tener ambos conceptos el conocimiento sobre un bien como base común, su propuesta aportó la base para estudios posteriores, los cuales sugerirán el papel moderador de familiaridad. El postulado primario sugiere que la familiaridad reduce el esfuerzo cognitivo que el individuo debe realizar en una decisión de consumo.

En cuanto a la destreza (expertise) como práctica del conocimiento, Alba y Hutchinson (1987) propusieron cinco 'dimensiones' de la misma. Es interesante detenerse en ellas, ya que ponen de manifiesto el vínculo entre la expertise y la familiaridad. Estas dimensiones son:

1. La simple repetición mejora el desempeño de una tarea al reducir el esfuerzo cognitivo que se requiere para ejecutarla.

2. Las estructuras cognitivas usadas para diferenciar los productos se afinan, se hacen más completas y verídicas conforme aumenta la familiaridad hacia ellos.

3. La habilidad de analizar la información, aislando lo que es más importante y relevante para la ejecución de las tareas, mejora el grado de familiaridad.

4. La habilidad de generar conocimiento preciso que va más allá del facilitado, sobre una determinada información, también mejora e incrementa la familiaridad.

5. La habilidad de recordar la información de los productos mejora conforme aumenta la familiaridad.

A lo largo de diversas publicaciones, durante la década de los ochenta, el término familiaridad fue hallando un espacio entre las variables observadas en los estudios de marketing y comunicación. Los trabajos precedentes -Krugman (1965), Zajonc (1968), Politz (1975), Park y Lessig (1977, 1981)- establecieron el terreno adecuado para acotar la familiaridad como una variable primordialmente cognitiva, y es en Baker, Hutchinson, et al. (1986) y Alba y Hutchinson (1987) donde se encuentra una definición del concepto en términos de las comunicaciones de marketing. 


\section{La familiaridad como variable moderadora de los procesos cognitivos}

Desde los años noventa en adelante, la perspectiva de la investigación se centró en validar y avanzar sobre las propuestas de los estudios precedentes. Sin embargo, el grueso del material de las publicaciones científicas se orientó hacia las primeras observaciones realizadas por Park y Lessig (1981) referidas a la función moderadora de la familiaridad.

Como se ha mencionado en el acápite anterior, la función moderadora se manifiesta de modo particular en los trabajos sobre el procesamiento de la información y las dinámicas cognitivas (ver Gill, 1988; Ambler, Braeutigam, et al., 2004; Ha, 2005).

Se comprende 'moderador' en el sentido usado en estadística: cuando en la relación entre dos variables se observa la participación de una tercera variable (en el marco de este estudio, la familiaridad). Las variables comprometidas en esta relación pueden ser cualitativas o cuantitativas, dependientes o independientes. (Para una exposición más detallada sobre el significado de una variable moderadora ver Baron y Kenny, 1986).

Kent y Allen (1994), Vanhuele (1999), y Kent y Kellaris (2001), fueron los primeros autores quienes estudiaron la interacción de la familiaridad (su acción como variable moderadora) en una dimensión propiamente cognitiva: en concreto su relación con la publicidad, la memoria y el comportamiento del individuo.

Los aportes de estos autores validaron las hipótesis sugeridas de que los consumidores procesan y codifican mejor la información nueva proveniente de productos familiares, aún en circunstancias de consumo de baja implicancia (low involvement) (Vargas, 2003).

A ello, agregaron que los recuerdos -el trabajo de rememoración- de la publicidad de marcas familiares se mantienen robustos aún ante la comunicación de marcas competidoras, en contraste con la situación de marcas poco familiares a una audiencia (ver también Tellis, 1997).

También en cuanto a la memoria, cabe mencionar lo registrado en un estudio por Dimofte y Yalch (2008), quienes argumentaron que la familiaridad participa moderando los procesos de rememoración tanto desde la memoria episódica (sucesos almacenados) como desde la memoria semántica (información almacenada).

Los investigadores Coates, Butler y Berry (2006) también registraron la participación de la familiaridad en la memoria implícita, comprendida como la rememoración involuntaria e inconsciente de la información previamente adquirida, efecto también conocido como priming. Los autores observaron una correlación positiva entre el fenómeno de priming y el grado de la familiaridad hacia las marcas. 
Por otra parte, Coates, Butler y Berry (2006) abordaron, aunque no de forma directa, el vínculo entre la familiaridad y la constitución de los conjuntos de consideración de las marcas que los conforman (para conjuntos de consideración ver Hauser, Toubia, et al., 2010).

De forma similar, el trabajo de Aurier, Jean y Zaichowsky (2000) reportó también una relación entre los conjuntos de consideración, el grado de familiaridad, y el contexto de consumo por parte del individuo. Los investigadores observan el impacto de la familiaridad del contexto, o circunstancia de consumo, sobre el tamaño del conjunto de consideración (número de marcas que integran dicho conjunto). Cabe notar la novedad de estos autores en aplicar el vocablo familiaridad a la circunstancia de consumo, ampliando su uso más allá del producto y la marca.

Por otro lado, los trabajos sobre la formación de actitudes y su influencia en el comportamiento del consumidor también hallaron en la familiaridad una variable de interés.

Los investigadores Phelps, Simonin y Ruth (1991), Campbell y Keller (2003) apoyaron los hallazgos de Machleit y Wilson (1988) y Dahlén y Lange (2004), quienes mostraron que los efectos de la publicidad en la actitud eran altos sobre los productos no-familiares y, por el contrario, no eran tan significativos en cuanto a las marcas familiares por parte de los individuos.

Una explicación parsimoniosa de este efecto, y concordante con las observaciones realizadas en el plano cognitivo expuestas en párrafos previos, es que en el caso del producto no-familiar el individuo cuenta con una estructura perceptual frágil, y en consecuencia más permeable a variabes externas (como el estímulo publicitario). En cuanto a las marcas familiares, éstas detentan un constructo cognitivo y perceptual, factor por el cual el influjo publicitario tiene menor impacto en la actitud formada hacia la marca.

Por su parte, Cox (1998), Laroche y Kim (1996) también han realizado estudios en los que argumentan la participación de la familiaridad en el proceso de formación y las dinámicas actitudinales. En ambos casos acordes con los autores ante citados y que también investigan en esta área del comportamiento del consumidor.

Por su parte, Hawkins y Hoch (2001) investigaron los efectos de la familiaridad y el procesamiento de la información con el efecto de la verdad (truth effect). Los autores sugirieron que mientras el procesamiento de información en alto grado de implicancia conlleva a una mejor retención en la memoria, el procesamiento en baja implicancia conduce a una retención más pobre pero de mayor creencia hacia la información procesada. Al mismo tiempo, argumentaron que los niveles de familiaridad median en el efecto de la verdad mientras los consumidores se encuentran expuestos a la información de marca. 
En cuanto a la familiaridad y los mensajes publicitarios, los estudios sobre el gusto/agrado hacia un anuncio (likeability) realizados por Rindfleisch e Inman (1998) y Du Plessis (1998), también utilizaron como base el procesamiento de información, utilizando el término familiaridad en un sentido más cercano a conocimiento.

Los citados autores sostuvieron que los anuncios y las marcas conocidas, y hacia las que tienen gusto/agrado, obtenían una mejor recordación y contaban con una mayor protección ante la publicidad de la competencia, en contraste con las marcas menos conocidas o familiares.

Por su parte, Hardesty, Carlson y Bearden (2002) argumentaron que el grado de familiaridad hacia una marca anunciada, moderaría el posible escepticismo hacia sus mensajes publicitario. Los autores sostuvieron que, así como una carencia de conocimiento del producto previene que los individuos tengan precios de referencia, un escenario donde la publicidad carece de credibilidad tampoco entregaría la información necesaria para valorar un producto.

Resultado congruentes se advierten en Lange y Dahlén (2003), así como en Dahlén y Lange (2004). Sus investigaciones sobre la congruencia/incongruencia en los mensajes publicitarios y la familiaridad de marca concluyen que, cuando la familiaridad de marca es baja, los mensajes publicitarios son proclives a obtener niveles de atención bajos por parte de los consumidores.

Otra manifestación de la función moderadora de la familiaridad ha sido reportada por Sun, Lin, et al. (2009) al estudiar su relación con el Efecto Dotación (Endowment Effect). De acuerdo con los autores, cuando el nivel de familiaridad hacia un producto es mayor, el Efecto-Dotación es menor. Los autores señalan que el fenómeno también ocurre a la inversa, cuando el nivel de familiaridad que se observa es bajo (para una exposición más detallada sobre el Efecto Dotación ver Kahneman, Knetsch, Thaler, 1991).

En paralelo con los estudios en torno a la familiaridad como un elemento moderador de las variables cognitivas, también han sido publicados diversos trabajos donde se exploran las relaciones entre la familiaridad y los diferentes efectos en el comportamiento del consumidor. Una de estas investigaciones es la de Sundram, Mitra y Webster (1998) sobre la valoración y la familiaridad de marca y la publicidad boca-a-boca.

La literatura cuenta también con publicaciones que tratan sobre el papel moderador de la familiaridad en la percepción de calidad. Se advierten los artículos de Lee y Ganesh (1999), quienes estudiaron el vínculo que existe entre la familiaridad del país de origen del producto y su calidad.

Moorthy y Zhao (2000), analizaron la familiaridad y su injerencia en el reconocimiento de marca y la sensibilidad hacia la percepción de calidad del producto. Son similares los estudios de Biswas (1992) y 
Vaydyanathan (2000) vinculados con la familiaridad y su influencia en los procesos de percepción y estimación del individuo respecto al precio de los productos.

\section{Conclusiones y estudios posteriores}

Parece razonable concluir tres observaciones generales luego de hacer una revisión histórica de la literatura.

En primer lugar, las teorías de mera exposición y procesamiento de información de baja implicación abordaron la comprensión de los efectos comunicacionales independientes del procesamiento activo de información. Este hecho facilitó el espacio para la observación de la familiaridad y la situó dentro del campo de los estudios cognoscitivos.

En segundo lugar, la aceptación de las definiciones sobre la familiaridad propuestas por Baker, Hutchinson, et al. (1986) y Alba y Hutchinson (1987), de modo general, hizo posible el establecimiento de un estándar entre la comunidad científica.

En tercer lugar, de forma congruente con las observaciones anteriores, es manifiesto que la familiaridad se considera como un factor participante en las dinámicas cognitivas del consumidor y, por consecuente, una variable moderadora en las diferentes etapas y manifestaciones del procesamiento de información y conocimiento del producto.

Por otro lado, se hallan pendientes investigaciones orientadas a clarificar ciertos aspectos del vocablo.

En este sentido, parece razonable hacer una crítica al supuesto de Baker, Hutchinson, et al. (1986) de que la familiaridad sea construcción unidimensional.

A lo largo de los últimos años, distintas investigaciones han reportado que la familiaridad tiene distintos componentes además del conocimiento, tales como las actitudes (Campbell y Keller, 2003; Machleit y Wilson, 1988; y las percepciones de atributos (Lee y Ganesh, 1999; Moorthy y Zhao (2000).

A pesar de tratarse de las variables vinculadas con el conocimiento, es plausible analizar estos factores para determinar qué tan válido es sostener que la familiaridad se trata de una construcción multi-dimensional.

Por último, a pesar de los estudios realizados sobre el objeto en cuestión, no se ha propuesto una metodología estructurada para la medición de la familiaridad. Este avance aseguraría la asequibilidad del concepto de familiaridad a la industria de la comunicación. De esta forma, las agencias de comunicaciones de marketing, así como los departamentos de marketing, encontrarían la familiaridad una herramienta métrica adicional a las existentes para conocer el estado de las marcas que gestionan. 


\section{Referencias bibliográficas}

Alba, J. W. y Hutchinson J. W. (1987): “Dimensions of consumer expertise”, Journal of Consumer Research, vol. 13, n. 4, pp. 411-545.

Ambler, T., Braeutigam, S., Stins, J., Rose, S., Swithenby, S. (2004): "Salience and choice: neural correlates of shopping decisions", Psychology and Marketing, 21(4), 247-261.

Aurier, P., Jean. S. y Zaichowsky, J.L. (2000): “Consideration set size and familiarity with usage context”, Advances in Consumer Research, 27, 307-313.

Baker, W., Hutchinson, W. J. Moore, D., Nedungadi, P. (1986): "Brand familiarity and advertising: effects on the evoked set and brand preference”, Advances in Consumer Research, vol. 13, Issue 1, pp. 637-643.

Baron, R. M. y Kenny, D. A. (1986): “The moderator-mediator variable distinction in social psychological research: Conceptual, strategic, and statistical considerations", Journal of Personality and Social Psychology, vol. 51(6), pp. 1173-1182.

Bettman, J.R. y Park C.W. (1980): "Effects of prior knowledge and experience and phrase of the choice process on consumer decision processes: a protocol analysis", Journal of Consumer Research, vol. 17, December, pp. 234-248.

Biswas, A. (1992): “The moderating role of brand familiarity in reference price perceptions”, Journal of Business Research, vol. 25, Issue 3, pp. 251-262.

Bogart, L. y Lehmam, C. (1973): "What makes a brand name familiar”, Journal of Marketing Research, vol. 10, February, pp. $17-22$.

Campbell, M. C. y Keller, K. (2003): "Brand familiarity and advertising repetition effects”, Journal of Consumer Research, vol. 30, Issue 2, pp. 292-304.

Coates, S., Butler, L. T., Berry, D. C., (2006): "Implicit memory and consumer choice: the mediating role of brand familiarity”, Applied Cognitive Psychology, vol. 20, pp.1101-1116.

Conover, J. N., (1982): "Familiarity and the structure of product knowledge", Advances in Consumer Research, vol. 9, pp. 494-498.

Cox, D. S., Cox, A. D. (1988): "What does familiarity breed? Complexity as a doderator of repetition effects in advertising evaluation”, Journal of Consumer Research, vol. 15, June, pp. 111-116.

Dahlén, M. y Lange, F. (2004): “To challenge or not to challenge: Ad-Brand incongruency and brand familiarity”, Journal of Marketing Theory and Practice, vol. 12, No. 3, pp. 20-35.

Dimofte, C. and Yalch, R. (2008): "The role of product category familiarity in self-referent advertising”, Advances in Consumer Research, vol. 35, pp. 726-727.

Du Plessis, E. (1998): “Memory and likeability: Keys to understanding and effects”, Admap, July/August, pp. 42-46. 
Gill, J. D., Grossbart, S., Laczniak, R. N. (1988): "Influence of involvement, commitment and familiarity on brand beliefs and attitudes of viewers exposed to alternative ad claim strategies", Journal of Advertising, vol. 17, No. 1, pp. 33-43.

Ha, Hong-Youl, (2005): "Effects of consumer perceptions of brand experience on the web: Brand familiarity, satisfaction and brand trust”, Journal of Consumer Behavior, vol. 4, pp. 438-452.

Hardesty, D.M. Carlson, J.P. y Bearden, W. O. (2002): "Brand familiarity and invoice price effects on consumer evaluations: the moderating role of skepticism toward advertising”, Journal of Advertising, vol. 31, No. 2, pp. 1-15.

Harrison, A.A. (1977): Mere exposure. En L. Berkowitz (Ed.). Advances in Experimental Social Psychology, Vol. 10, New York. King's Crown.

Hauser, J. R., Toubia, O., Evgeniou, T., Bufurt, R., Dzyabura, D. (2010): “Disjunctions of conjunctions, cognitive simplicity, and consiedarions sets", Journal of Marketing Research, vol. XLVII, June, pp. 485-496

Hawkins, S. A. y Hoch, S. J. (1992): “Low-Involvement learning: memory without evaluation”, Journal of Consumer Research, December, vol. 19, pp. 221-225.

Hawkins, S. A. Hoch, S. J. y Meyers-Levy. J. (2001): “Low involvement learning: repetition and coherence in familiarity and belief”, Journal of Consumer Psychology, vol. 11, Issue, 1, pp. 1-11.

Howard, J.A., Sheth, J.N. (1969): The Theory of Buying Behaviour. N.Y.: John Wiley \& Sons Inc.

Jacoby, L.L. (1991): “A process dissociation framework: Separationg automatic from intentional uses of memory”, Journal of Memory and Language, vol. 30, pp. 513-541.

Johnson, E. J. y Russo, E. J., (1984): “Product familiarity and learning new information”, Journal of Consumer Research, June, vol. 11, pp. 542-550.

Kahneman, D., Knetsch, J. L.. Thaler, R. H. (1991): “Anomalies: The Endowment Effect, Loss Aversion, and Status Quo Bias”, Journal of Economic Perspectives, vol. 5, No. 1, pp. 193-206.

Kent, R, J., y Kellaris, J. J. (2001): “Competitive interference effects in memory for advertising: are familiar brands exempt?”, Journal of Marketing Communications, vol. 7, No. 3, pp. 159-169.

Kent, R. J. y Allen, C. T. (1994): "Competitive interference effects in consumer memory for advertising the role of brand familiarity”, Journal of Marketing, July, vol. 58, pp. 97-105.

Krugman, H. E. (1965): “The impact of television adversiting: Learming without involvement”, Public Opinion Quarterly, vol. 29, pp. 439-356.

Krugman, H. E. (2000): "Memory without recall, exposure without perception”, Journal of Advertising Research, vol. 17, pp. 7-12.

Lange, F. y Dahlen, M. (2003): "Let's be strange: brand familiarity and ad-brand incongruency", Journal of Product and Brand Management, vol. 21, Issue 7, pp. 449-461. 
Laroche, M., Kim, Ch., Zhou, L. (1996): "Brand familiarity and confidence as determinants of purchase intention: an empirical test in multiple brand context”, Journal of Business Research, vol. 37, Issue 2, pp. 115-120.

Lee, D. y Ganesh, G. (1999): "Effects of partitioned country image in the contex of brand image and familiarity. A categorization theory perspective", International Marketing Review, vol. 16, No. 1, pp. 1-4.

Machleit, K. A. y Wilson, D. A. (1988): "Emotional feelings and attitude toward the advertisement: the roles of brand familiarity and repetition", Journal of Advertising, vol. 17, No. 3, pp. 27-35.

Marks, L. J. y Olson, J. C. (1981): “Toward a cognitive structure conceptulization of product familiarity”, Advances in Consumer Research, vol. 8, Issue 1, pp. 145-150.

Monroe, K. B. (1976): “The influence of price differences and brand familiarity on brand preferences", Journal of Consumer Research, vol. 3, No. 1, pp. 42-49.

Moothy, S., y Zhao, H. (2000): “Advertising spending and percieved quality”, Marketing Letters, vol. 11:3, pp. 221-233.

Park, C. W. y Lessig V. P. (1981): "Familiarity and its impact on consumer decision biases and heuritics", Journal of Consumer Research, vol. 8, pp. 223-230.

Park, C. W. y Lessing, V. P. (1977): “Judgmental rules and stage of the familiarity curve: promotional implications”, Journal of Advertising, vol. 6, Issue 1, pp. 10-16.

Phelps, J. y Thorson, E. (1991): "Brand familiarity and product involvement effects on the attitude toward an ad brand attitude relationship", Advances in Consumer Research, vol. 18, pp. 202-209.

Politz, A. (1975): "The function of advertising and its measurements", Journal of Advertising, vol. 4, Issue 2, pp. 10-12.

Rindfleisch, A., Inman, J. J. (1988): "Explaining the familiarity-Liking relationship: mere exposure, information availability, or social desirability?", Marketing Letters, vol. 9, No. 1, pp. 5-19.

Simonin, B. L., Ruth, J. A. (1998): "Is a company known by the company it keeps? Assessing the spillover effects of brand alliances on consumer brand attitudes", Journal of Marketing Research, February, vol. XXXV, pp. 30-42.

Sun, Ya-Chung, Lin, Chien-Huang, Cheng, Yin-Hui y Chuang, Shih-Chieh (2009): "The moderating effect of product familiarity on the endowment effect", Advances in Consumer Research, vol. 36, pp. 974-975.

Sundaram, D.S. Mitra, K. y Webster, C. (1998): "Word of mouth comunications: a motivational analysis", Advances in Consumer Research, vol. 25, pp. 527-531.

Tellis, G. J. (1997): “Effective frequency: one exposure or three factors?”, Journal of Advertising Research, vol. 37, Issue 4, pp. 7-12.

Vanhuele, M. (1995): "Why familiar stimuli are better liked. A study on the cognitive dynamics linking recognition and mere exposure effect”, Advances in Consumer Research, vol. 22, pp. 171-175. 
Vargas, Lizardo (2003): "Procesamiento de infomación y familiaridad de marca”, Análisi Cuaderns de Comunicació i Cultura, vol. 30, pp. 225-240.

Vaydyanathan, R. (2000): “The Role of Brand Familiarity in Internal Reference Price Formation: an Accessibility-Diagnosticity Perspective”, Journal of Business and Psychology, vol. 14, No. 4, pp. 605-624.

Zaichowsky, L. L. (1985): "Familiarity: product use, involvement or expertise”, Advances in Consumer Research, vol. 12, pp. 296-299.

Zajonc, R. B., (1968): "Attitudinal effects of mere exposure", Journal of Personality and Social Psychology. Monograph Supplement, vol. 9, No., 2 part 2, pp.1-27. 
